\title{
CURING PROCESS OF BENZOXAZINE SYSTEMS. AN EXPERIMENTAL AND THEORETICAL STUDY.
}

\author{
E. GILBERT ${ }^{\dagger}$, A. FORCHETTI ${ }^{\dagger}$, J.I. PESOA ${ }^{\dagger}$, E. BERKENWALD ${ }^{\dagger}$, \\ M. SPONTÓN ${ }^{\dagger}$ and D. ESTENOZ \\ $\dagger$ INTEC (Universidad Nacional del Litoral - CONICET) - Güemes 3450 - (3000) Santa Fe - Argentina. \\ destenoz@santafe-conicet.gov.ar \\ † ITBA (Instituto Tecnológico Buenos Aires) - Av. Madero 399 - (1106) Buenos Aires - Argentina.
}

\begin{abstract}
A mathematical model that simulates the curing process of benzoxazine $(\mathrm{Bz})$ systems is presented. The model predicts the conversion, gel point and $\mathrm{Tg}$ along the curing process, and considers the diffusional limitations to mass transfer due to the increase in the system viscosity along the process. This model can be used to select an appropriate combination of time and temperature in order to obtain a material with pre-specified properties. The theoretical parameters were adjusted with experimental data: conversion, weight-average molecular weight, weight fraction of solubles and $\mathrm{Tg}$. The Bz based on bisphenol $A$ and aniline (BzBA) was used to adjust the model. The curing kinetic of this Bz was followed by FTIR, SEC and DSC, considering five different curing conditions. A very good agreement between experimental and simulated values was observed, even when curing is carried out under different temperatures profiles.
\end{abstract}

Keywords: Benzoxazine, Curing, Polybenzoxazine, Polymer, Properties,

\section{INTRODUCTION}

Polybenzoxazines (PBz) are obtained by thermally activated ring-opening polymerization of benzoxazine $(\mathrm{Bz})$ monomers, following a cationic mechanism without generating any by-products (Burke et al., 1965; Ma et al., 2017). It has been reported (Dunkers et al., 1996; Ghosh et al., 2007) that the conformation of an oxazine ring attached to a benzene ring could be a distorted semichair structure, with the nitrogen and the carbon atoms between the nitrogen and the oxygen atoms placed above and below the benzene ring plane, respectively. This preferential conformation makes the oxygen and the nitrogen atoms of the oxazine ring potential sites of cationic polymerization initiation, leading to the formation of a phenoxy-type Mannich base (Wang and Ishida, 2000). Several authors (Liu and Gu, 2001; Chutayothin and Ishida, 2010) predict that the oxygen might be the preferred site over nitrogen due to its high negative charge. The ortho position of the benzene ring with respect to the phenoxy group is another potential site of cationic polymerization. Therefore, it is reasonable to assume that, upon initiation, propagation can also proceed by insertion of the monomers via the reaction of the free benzene ortho position, generating a phenolic-type Mannich base polymer. It was also reported (Wang and Ishida, 2000) that the PBz structures obtained via thermal curing could follow the phenolic-type mechanism, generating a phenolic Mannich bridges network.

According to several authors (Ning and Ishida, 1994), the ring opening reactions at high temperatures generate free phenolic groups that accelerate ring opening. Thus, thermal polymerization of Bzs could proceed via an autocatalytic mechanism where phenolic groups formed at the beginning of the heating promote $\mathrm{Bz}$ ring opening and accelerate the process due to their acid character (Hamerton et al., 2012). Despite this autocatalytic effect, the polymerization of $\mathrm{Bz}$ usually requires high temperatures, above $180{ }^{\circ} \mathrm{C}$ (Ishida and Rodriguez, 1995). In consequence, the polymerization can often overlap with the initio of the degradation (Ishida and Ohba, 2005).

The processability and final properties of a thermosetting resin are critically dependent on the rate and extent of cure. Curing is a complex process that includes gelation, vitrification and subsequent crosslinking, obtaining a three-dimensional network (He et al., 2013).

$\mathrm{PBz}$ curing processes were investigated in several publications (Ishida and Froimowicz, 2017). The main method applied to the study of PBz curing kinetics and to obtaining kinetic parameters is the isoconversional method by DSC (Jang and Shin, 1995; Rucigaj et al., 2015; Ishida and Rodriguez, 1995). Spontón et al. (2012) applied statistical models to the FTIR data obtained during the homopolymerization (at $180^{\circ} \mathrm{C}$ ), showing that the Bz derived from bisphenol A, hereafter BzBA, exhibits a unique chemical reaction forming a network of phenolic Mannich bridges. The experimental data are in good agreement with the model results at the early stages of curing. However, at a later stage, particularly near vitrification, the reaction becomes primarily diffusion controlled, which results in deviations between experimental and theoretical data (Rishwana et al., 2016). Wang et al. (2016) investigated the curing process by DMTA at different temperatures, using isothermal processes between 200 and $220 \mathrm{C}$. The results showed conversion values at the gel point in the range of $37.9 \%$ to $42.8 \%$.

The study of the curing reaction of Bzs is a very interesting area, since there are still unresolved questions regarding crucial aspects, such as the relationship between process conditions, curing degree and final material properties. These are key aspects to elucidate in order to control and optimize process conditions and performance of final products. While several thermosetting systems have been theoretically studied, there are no models 
available in the literature for Bzs polymerization capable of adequately estimating the evolution of the main reaction variables, as well as the molecular structure.

In this article, a detailed study of the curing of BzBA is presented. An extensive experimental work combined with a mathematical model is developed with the aim of determining the main characteristics of the polymer network as a function of the curing conditions.

\section{METHODS}

\section{A. Materials}

The following chemical reagents were purchased from the sources indicated: bisphenol A ( $\geq 97 \%$, Aldrich), aniline $(\geq 99.5 \%)$ from Merck; $N, N$-dimethylformamide from Dorwil; paraformaldehyde $(\geq 85 \%)$, methylene chloride $(\geq 99.5 \%)$ and sodium hydroxide $(\geq 97 \%)$ from Cicarelli.

\section{B. Synthesis and Characterization of BzBA}

The synthesis of BzBA was carried out using a two-stage procedure (Brunovska et al., 1999) from bisphenol A, aniline, and paraformaldehyde.

The BzBA structure was analyzed by ${ }^{1} \mathrm{H}$ and ${ }^{13} \mathrm{C}$ NMR and FTIR. ${ }^{1} \mathrm{H}$ NMR and ${ }^{13} \mathrm{C}$ NMR in deuterated DMSO were registered on a Bruker 300 Ultrashield (300 MHz) NMR spectrometer with Fourier transform. The ${ }^{1} \mathrm{H}$ NMR spectrum indicated the presence of different aromatic protons at $7.32(2 \mathrm{H}, \mathrm{Ar}), 7.15(4 \mathrm{H}, \mathrm{Ar}), 6.90$ $(6 \mathrm{H}, \mathrm{Ar}), 6.86(2 \mathrm{H}, \mathrm{Ar})$ and $6.74 \mathrm{ppm}(2 \mathrm{H}, \mathrm{Ar})$. The characteristics peaks of the oxazine ring were also identified at $5.28\left(4 \mathrm{H}, \mathrm{O}-\mathrm{CH}_{2}-\mathrm{N}\right)$ and $4.66 \mathrm{ppm}\left(4 \mathrm{H}, \mathrm{C}_{\mathrm{Ar}}-\mathrm{CH}_{2}-\mathrm{N}\right)$. The methylene protons were identified at $1.63 \mathrm{ppm}(6 \mathrm{H}$, $\mathrm{C}-\mathrm{CH}_{3}$ ). No signal corresponding to ring opening was observed, revealing the purity of the Bz. In accordance with these results, the ${ }^{13} \mathrm{C}$ NMR spectrum also showed the existence of different aromatic carbons by the presence of the peaks at $152.5,149.0,143.5,129.1,126.5,124.9$, $121.5,120.1,118.2,116.6 \mathrm{ppm}$; methylene carbons at $31.2 \mathrm{ppm}$ and the existence of the oxazine ring at 78.9, 50.7 and 41.9 ppm. FTIR spectra were obtained using a Perkin Elmer FTIR spectrophotometer Spectrum One model with transmission accessories. For this analysis, a fine suspension of the samples was dispersed in $\mathrm{KBr}$, and then pressed to form transparent $\mathrm{KBr}$ pellets. The FTIR spectrum showed the characteristics bands of the Bzs at 945 and $1500 \mathrm{~cm}^{-1}$ associated to the oxazine ring and to the tri-substituted benzene ring, respectively (Shukla $e t$ al., 2016; Han et al., 2017). A further band of interest was identified at $752 \mathrm{~cm}^{-1}$ attributed to the $\mathrm{N}-\mathrm{C}_{\mathrm{Ar}}$ stretching (Soto et al., 2016).

\section{Crosslinking Reactions and Characterization}

BzBA curing reactions were monitored by dynamic DSC, using a Mettler DSC821e thermal analyzer under nitrogen as a purge gas $\left(20 \mathrm{~mL} \mathrm{~min}^{-1}\right)$ and a heating rate

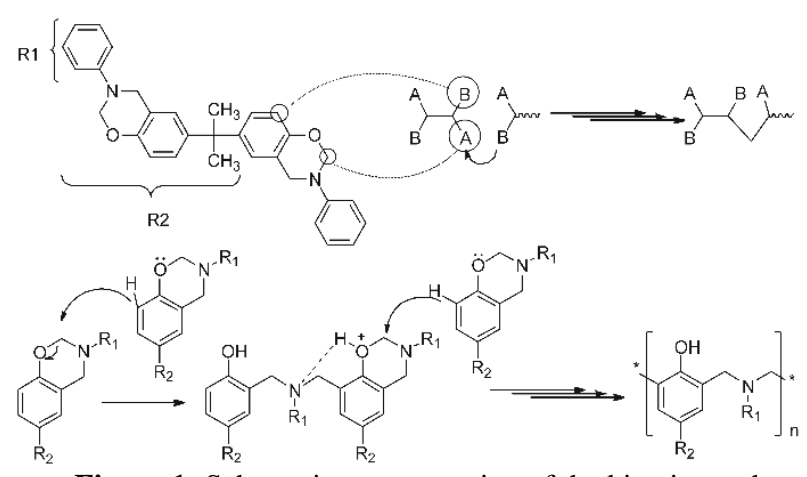

Figure 1: Schematic representation of the kinetic mechanism adopted; $A$ and $B$ represent the oxazine ring and the hydrogen in ortho position, respectively.

of $10 \mathrm{Cmin}^{-1}$. As it is well known (Jubsilp et al. 2006), BzBA exhibited one dominant autocatalytic curing process that involves oxazine ring opening forming a threedimensional network of phenolic Mannich bridges (Fig. 1).

The curing kinetic was studied considering the following conditions: $\mathrm{CC} 1\left(160^{\circ} \mathrm{C}\right.$ for $\left.5 \mathrm{~h}\right), \mathrm{CC} 2(180 \mathrm{C}$ for $5 \mathrm{~h}), \mathrm{CC} 3\left(110^{\circ} \mathrm{C}\right.$ for $2 \mathrm{~h}, 120 \mathrm{C}$ for $1 \mathrm{~h}$ and $150^{\circ} \mathrm{C}$ for $\left.2 \mathrm{~h}\right)$; CC4 (160 C for $2 \mathrm{~h}, 180 \mathrm{C}$ for $1 \mathrm{~h}$ and $190^{\circ} \mathrm{C}$ for $2 \mathrm{~h}$ ); CC5 $\left(180^{\circ} \mathrm{C}\right.$ for $2 \mathrm{~h}, 200^{\circ} \mathrm{C}$ for $1 \mathrm{~h}$ and $215^{\circ} \mathrm{C}$ for $\left.2 \mathrm{~h}\right)$. Curing conditions $\mathrm{CC} 1$ and $\mathrm{CC} 2$ involve isothermal curing while $\mathrm{CC} 3, \mathrm{CC} 4$ and CC5 involve different temperature profiles. To follow the progress of the curing reaction, several samples were taken at different times and analyzed by FTIR and DSC. The weight fraction of solubles $\left(W_{\text {Sol }}\right)$ was determined for all collected samples by an extraction process using $\mathrm{N}, \mathrm{N}$-dimethylformamide. The soluble fraction was isolated. The weight-average molecular weights $\left(M_{w}\right)$ were determined by SEC up to the gel point using a Waters chromatograph equipment with a Waters 1525 pump, a Shodex KD-803 column $(8 \times 300 \mathrm{~mm})$ and a Waters 2412 refractive index detector using $N, N$-dimethylformamide as eluent, with a flow rate of $1 \mathrm{mLmin}^{-1}$ at 40 C. Polyethylene glycol (PEG) standards (pegkitwa07) were used for calibration. The insoluble solid was washed with water and methylene chloride, dried under vacuum during $24 \mathrm{~h}$ and characterized gravimetrically.

The conversion $(x)$ was determined from FTIR analysis, following the reduction of the peak at $945 \mathrm{~cm}^{-1}$ associated to the $\mathrm{Bz}$ ring. To this effect, the absorbance intensity was normalized with respect to the unalterable band at $752 \mathrm{~cm}^{-1}$ (Spontón et al., 2012). FTIR spectra of BzBA (Fig. 2.a-e) show the progressive decrease with time of the characteristic band at $945 \mathrm{~cm}^{-1}$ indicating ring-opening. As a consequence of this ring-opening, a progressive diminution of the band at $1500 \mathrm{~cm}^{-1}$, corresponding to the vibration of tri-substituted benzene ring, and the development of the band at $1490 \mathrm{~cm}^{-1}$ corresponding to the tetra-substituted benzene ring can also be observed (Gârea et al., 2007). The development of bands at 1620, 2960 and $3380 \mathrm{~cm}^{-1}$ is due to the increase of aliphatic methylene groups and phenolic groups (Li et al., 2017). These results thus confirm the formation of 

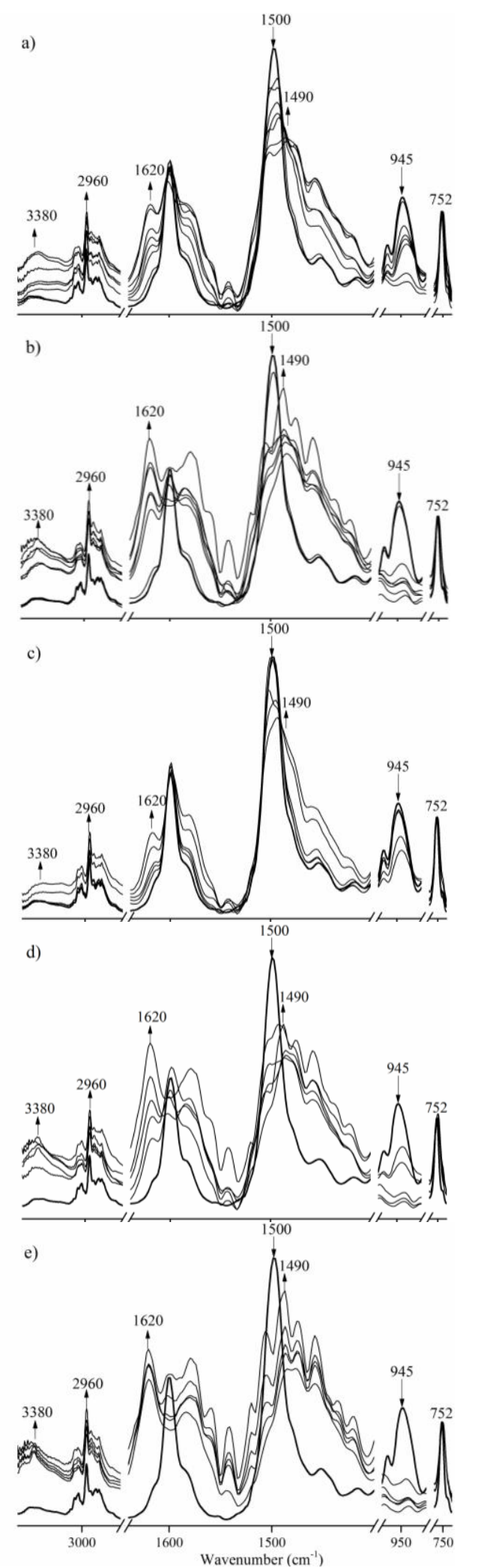

Fig. 2: FTIR spectra of BzBA curing process under isothermal conditions: a) $\mathrm{CC} 1$ and b) $\mathrm{CC} 2(0,6,32,45,60,75,180$, and $315 \mathrm{~min}$ ); and under different temperature profiles: c) CC3, d)

CC4, and e) CC5 (0, 65, 125, 190, 255, and $315 \mathrm{~min})$.

phenolic Mannich bridges and the consequent formation of a crosslinked network by homopolymerization (see Fig. 1).

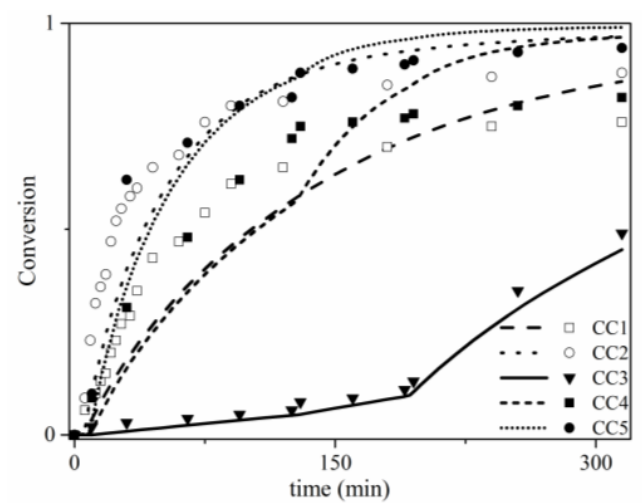

Fig. 3: Evolution of experimental data (in symbols) and theoretical results (in lines) for conversion with time.

In Fig. 3, the evolution of conversion with time under different curing conditions is shown. For CC1 and CC4 experiments, carried out at the same temperature until $125 \mathrm{~min}$ (both at $160 \mathrm{C}$ ), it can be observed that the $\mathrm{Bz}$ reacts very fast at the beginning of the process. However, at the end of the process, a final conversion of 0.82 was obtained for the CC4 experiment, while for the CC1 experiment carried out under isothermal conditions, the obtained conversion was lower. Regarding CC2 and CC5 experiments, the conversions were similar (around 0.8) until 120min. However, for CC5 experiment the temperature was then raised up to $215^{\circ} \mathrm{C}$ and the final conversion was 0.94 , while for the isothermal curing at $180^{\circ} \mathrm{C}$ the final conversion was 0.88 . Finally, for CC3 experiment, the conversion reached at the end of the process was 0.49 .

Figure 4 shows the evolution of $M_{w}$ up to the gel point and $W_{S o l}$ thereafter, for the experiments carried out under different curing conditions. For CC3 and CC4 experiments, the curing does not appear to be complete at the end of the curing processes. Moreover, at the end of CC3 experiment, a high concentration of soluble unreacted monomers and oligomers was obtained. In contrast, for CC5 experiment, carried out at higher temperature, the curing was practically completed and no $W_{\text {Sol }}$ was observed at the end of the curing process. The experimental results indicate that the gel point occurs at a conversion between 0.32 and 0.35 . Moreover, gel times for the CC1 and CC2 experiments are 32-36 and 12-15min, respectively.

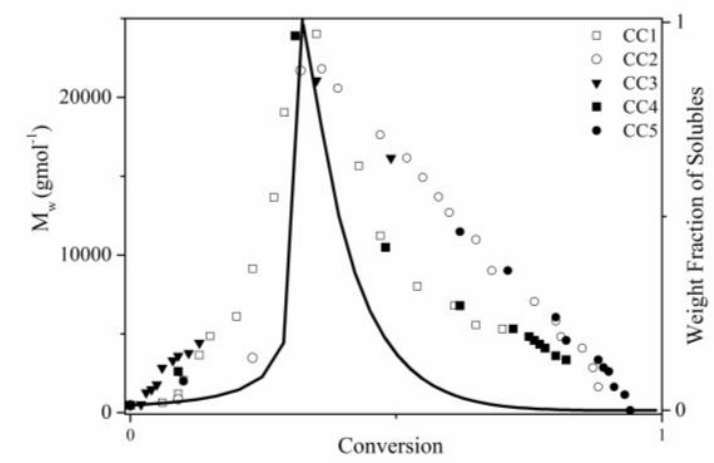

Fig. 4: Evolution of experimental data (in symbols) and theoretical results (in lines) for $M_{w}$ and $W_{\text {sol }}$ as functions of conversion. 


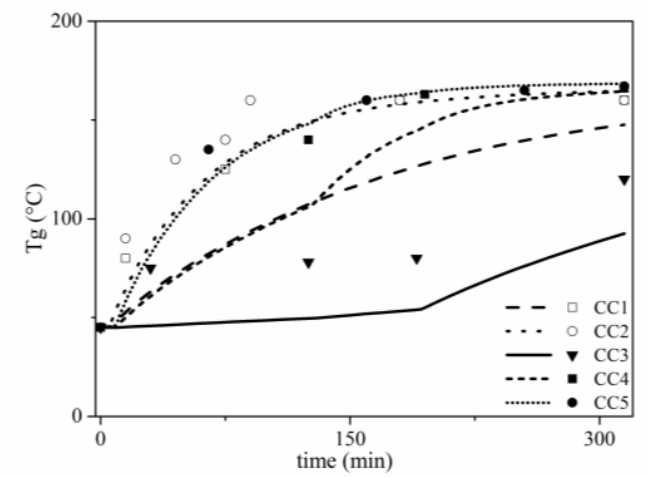

Fig. 5: Experimental data (in symbols) and theoretical results (in lines) for $T g$ as a function of time.

In addition, samples taken at different times were also characterized by DSC to study the evolution of its $T g$ during the different curing processes. The results of these measurements are summarized in Fig. 5. Note that the $T g$ increase with time and curing temperature. For CC3 experiment, after $300 \mathrm{~min}$ a $\mathrm{Tg}$ of $120 \mathrm{C}$ was obtained. As expected, by increasing the curing temperature the crosslinking density of the material increases (Gilbert et al., 2018).

\section{Kinetic Mechanism}

The mathematical modeling of $\mathrm{Bz}$ curing is based on a kinetic mechanism that assumes that secondary reactions (such as the formation of phenoxy-type Mannich bases, methylene bridges and reactions in the position ortho and para of benzene-amine) and shrinkage or volumetric expansion upon curing are negligible (Ishida and Low, 1997). The schematic representation of the kinetic mechanism adopted is shown in Scheme 1, where $A$ and $B$ represent the oxazine ring and the hydrogen in the ortho position, respectively.

The developed mathematical model is based on the curing kinetics combined with an extension of the technique proposed by Macosko and Miller (1976), and Miller and Macosko $(1976,1978)$ for a stepwise nonlinear polymerization and postgel properties estimation. The model consists of two interdependent modules: the Global Module, predicting the evolution of conversion with reaction time; and the Network Properties Module, based on the recursive nature of the curing reaction as well as laws of probability and expectation, which quantifies the evolution of $M_{w}, W_{S o l}$ and gel point; and predicts the $T g$ of the cured resin. The mathematical model allows relating the time of cure to the main reaction variables, as well as PBz molecular characteristics and physical properties.

The Global Module is based on the mass balances for the reacting functional groups and calculates the evolution of the chemical species concentrations with curing time. Since both $A$ and $B$ are always present in equal amounts and taking into consideration the second order kinetic mechanism adopted, the mass balance expression for functional groups became:

$$
\frac{d[A]}{d t}=-k[A]^{2}
$$

In order to consider diffusional effects along the curing process, an expression proposed by Hui and Hamielec (1972) is adopted, where $A_{0}$ and $E_{a}$ are respectively the Arrhenius pre-exponential factor and activation energy, $R$ is the universal gas constant, $T$ is curing temperature and $x$ is the conversion.

$$
k(T, x)=A_{0} \exp \left(-E_{a} / R T\right) \phi(T, x)
$$

The factor $\phi$ accounts for the diffusional effects associated to the increase in system viscosity due to crosslinking. This factor is related to conversion and temperature using a gel-effect type expression:

$$
\phi(x, T)=\exp \left(-\left(C_{1}(T) x+C_{2}(T) x^{2}+C_{3}(T) x^{3}\right)\right.
$$

The fraction of reacted functional groups at any time $t$ during the curing process, related to the conversion are calculated with

$$
x(t)=\frac{[A]_{0}-[A]}{[A]_{0}},
$$

where the subscript $O$ denotes the initial concentrations.

The Network Properties Module estimates the network properties based on the works of Miller and Macosko $(1976,1978)$, supposing that a monomer cures up to a certain conversion, related to the fraction of reacted functional groups $\left(x_{(t)}\right)$. The conversion is directly related to the reaction time by means of the Global Module. For a randomly selected $A$ in the network, the recursive nature of the curing can be combined with the laws of total probability to evaluate the average molecular characteristics of the surrounding polymer network.

The $M_{w}$ of the network polymer can be estimated with (Macosko and Miller, 1976):

$$
\bar{M}_{w}=M \frac{1+x}{1-x\left(f_{e}-1\right)},
$$

where $M$ is the molar mass of the monomer and $f_{e}$ is an equivalent monomer functionality with a value of 4 . The evolution of $M_{w}$ can thus be related to curing time.

When the curing system reaches the gel point, a structure with an infinite molecular weight is obtained ( $\mathrm{gel}$ ). The fraction of monomer and lower molecular weight polymer $\left(W_{\text {Sol }}\right)$ remains in the soluble fraction $($ sol $)$. Up to the gel point, all the polymer molecules are finite; therefore, the $W_{S o l}$ is unity. Beyond the gel point, the rapid incorporation of molecules into the network causes its value to decrease. A randomly chosen group of a molecule will be part of the sol if all of its arms lead out to finite chains. Then, the $W_{\text {Sol }}$ can be estimated as (Miller and Macosko, 1976):

$$
W_{\text {Sol }}=\left[p\left(F_{A}\right)\right]^{f_{e}}=\left[\left(\frac{1}{x}-\frac{3}{4}\right)^{1 / 2}-\frac{1}{2}\right]^{f_{e}},
$$

where $p\left(F_{A}\right)$ is the probability that the randomly chosen A group leads to a finite chain.

The $T g$ is calculated from a modified Di Benedetto (1987) equation for the case of a thermosetting polymer:

$$
\frac{T_{g}-T_{g 0}}{T_{g \infty}-T_{g 0}}=\frac{\lambda x(t)}{1-(1-\lambda) x(t)},
$$

where $T g_{\infty}$ and $T g_{0}$ are the $T g$ of the completely reacted system and the monomer, respectively and $\lambda$ is a parameter adjusted with a value of 0.75 . 


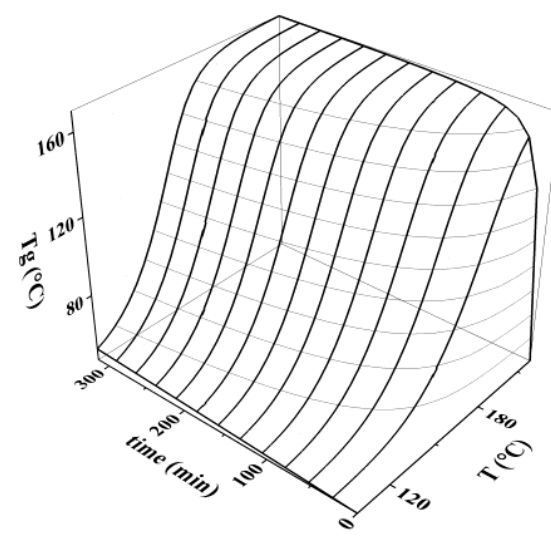

Fig. 6: Predicted $T g$ as a function of time and isothermal curing temperature.

\section{E. Simulations, Results and Discussion}

The mathematical equations from the different modules were programmed in MATLAB v8.3. The systems of ordinary differential equations are solved using standard Runge-Kutta algorithms, while algebraic equations are solved using routines based on Newton-Raphson methods. A typical simulation requires about $6 \mathrm{~s}$ on an Intel iCore 5 processor at $2.93 \mathrm{GHz}$ and 6GB RAM.

The model parameters were adjusted with the experimental data: conversion, $M_{w}, W_{\text {sol }}$ and $T g$. The $\phi$ factor was adjusted as: $C_{l}=2.57-5.05 \times 10^{-3} \times T ; \quad C_{2}=9.56$ $1.76 \times 10^{-2} \times T$ and $C_{3}=-3.03+7.85 \times 10^{-3} \times T$. The $E_{a}$ and $A_{0}$ values adopted for the Arrhenius expression (Eq. 2) were $84 \mathrm{KJ} \mathrm{mol}^{-1}$ and $1.77 \times 10^{-2} \mathrm{~min}^{-1}$, respectively. A very good agreement between experimental and simulated conversion values is observed, even when curing is carried out under different temperatures profiles. For CC1 experiment, the estimated theoretical conversion is higher than the experimental value (Fig. 3). This can be explained by considering that while curing proceeds, the $\mathrm{Tg}$ of the system increases (Fig. 5). Once $T g$ reaches the curing temperature, vitrification takes place, and diffusion begins to control the curing process thereafter.

Regarding the evolution of $M_{w}$, the experimental data show a good agreement with theoretical calculations (Fig. 4). The estimated $W_{\text {sol }}$ are smaller than expected due to experimental errors associated to the detection limit of the equipment. However, a good agreement between estimated and simulated conversion values at the gel point is observed. These values indicate that the gel point occurs at a conversion of around 0.33 . In addition, this value is in agreement with the reported in the literature (Wang et al. 2016).

Finally, as it is shown in Fig. 6, the model was also used to predict the effect on the Tgs of curing time and temperature of materials in the range of the experimental variables used for the model adjustment. Different combinations of time and temperature can be selected to obtain a material with a determined $\mathrm{Tg}$ for a particular propose.

\section{CONCLUSIONS}

The curing process of BzBA was experimentally and theoretically investigated using different curing conditions.
The experiments $\mathrm{CC} 1$ and $\mathrm{CC} 2$ were performed at constant temperature. Experiments CC3, CC4 and CC5 were carried out under different curing profiles. The evolution of conversion, $W_{s o l}, M_{w}$ and $T g$ were determined for all experiments.

For the theoretical work, a mathematical model was developed to simulate the curing process and determine kinetic parameters. A very good agreement between experimental and simulated values is observed.

The model predicts the evolution of the molecular structure of the polymer and the gel point. After gelation, the evolution of $W_{\text {sol }}$ is related to the increase of crosslinking density, which is predicted by the model. In addition, the model was also adjusted to predict the evolution of $T g$ of the system during the curing under different conditions.

The model can also be used, in the range of experimental variables studied, to select an appropriate combination of time and curing temperature in order to obtain a material with pre-specified $T g$.

\section{REFERENCES}

Brunovska, Z., Liu, J.P. and Ishida, H. (1999). “1,3,5triphenylhexahydro-1,3,5-triazine - active intermediate and precursor in the novel synthesis of benzoxazine monomers and oligomers," Macromol. Chem. Phys., 200, 1745-1752.

Burke, W.J., Bishop, J.L., Glennie, E.L.M. and Bauer, W.N. (1965). "A new aminoalkylation reaction. condensation of phenols with dihydro-1,3aroxazines," J. Org. Chem., 30, 3423-3427.

Chutayothin, P. and Ishida, H. (2010). "Cationic ringopening polymerization of 1,3-benzoxazines: mechanistic study using model compounds," Macromol., 43, 4562-4572

Di Benedetto, A.T. (1987). "Prediction of the glass transition temperature of polymers: a model based on the principle of corresponding states," J. Polym. Sci. Part B Polym. Phys., 25, 1949-1969.

Dunkers, J., Zarate, E.A. and Ishida, H. (1996). “Crystal structure and hydrogen-bonding characteristics of $\mathrm{N}, \mathrm{N}$-bis (3,5-dimethyl-2-hydroxybenzyl) methylamine, a benzoxazine dimer," J. Phys. Chem., 100, 13514-13520.

Gârea, S.A., Iovu, H., Nicolescu, A. and Deleanu, C. (2007). "Thermal polymerization of benzoxazine monomers followed by GPC, FTIR and DETA," Polym. Test., 26, 162-171

Ghosh, N.N., Kiskan, B. and Yagci, Y. (2007). "Polybenzoxazines-New high performance thermosetting resins: synthesis and properties," Prog. Polym. Sci., 32, 1344-1391.

Gilbert, E., Taverna, M.E., Dieser, M.F., Morales, G., Spontón, M. and Estenoz, D. (2018). "Synthesis and characterization of new thermosetting polybenzoxazines with other functional groups in the network," J. Polym. Res., 25, 114-123.

Hamerton, I., Howlin, B.J., Mitchell, A.L., McNamara, L.T. and Takeda, S. (2012). "Systematic examination of thermal, mechanical and dielectri- 
cal properties of aromatic polybenzoxazines," $R e$ act. Funct. Polym., 72, 736-744.

Han, L., Iguchi, D., Gil, P., Heyl, T., Sedwick, V.M., Arza, C.R., Ohashi, S., Lacks, D.J. and Ishida, H. (2017). "Oxazine ring-related vibrational modes of benzoxazine monomers using fully aromatically substituted, deuterated ${ }^{15} \mathrm{~N}$ isotope exchanged, and oxazine-ring-substituted compounds and theoretical calculations," J. Phys. Chem. A., 121, 62696282.

He, X.Y., Wang, J., Wang, Y.D., Liu, C.J., Liu, W.B. and Yang, L. (2013). "Synthesis, thermal properties and curing kinetics of fluorene diamine-based benzoxazine containing ester groups," Eur. Polym. J., 49, 2759-2768.

Hui, A.W. and Hamielec, A.E. (1972). "Thermal polymerization of styrene at high conversions and temperatures," J. Appl. Polym. Sci., 16, 749-769.

Ishida, H., and Froimowicz, P. (2017). Advanced and Emerging Polybenzoxazine Science and Technology, Elsevier.

Ishida, H. and Low, H.Y. (1997). "A study on the volumetric expansion of benzoxazine-based phenolic resin," Macromol., 30, 1099-1106.

Ishida, H. and Ohba, S. (2005). "Synthesis and characterization of maleimide and norbornene functionalized benzoxazines," Polymer, 46, 5588-5595.

Ishida, H. and Rodriguez, Y. (1995). "Curing kinetics of a new benzoxazine-based phenolic resin by differential scanning calorimetry," Polymer, 36, 3151-3158.

Jang, J. and Shin, S. (1995). "The kinetics of B-a and Pa type copolybenzoxazine via the ring opening process," Polym. J., 27, 601-606.

Jubsilp, C., Damrongsakkul, S., Takeichi, T. and Rimdusit, S. (2006). "Curing kinetics of arylaminebased polyfunctional benzoxazine resins by dynamic differential scanning calorimetry," Thermochim. Acta, 447, 131-140.

Li, S., Yang, C., Li, C. and Yan, S. (2017). "Synthesis, characterization of new bisphenol-based benzoxazines and the thermal properties of their polymers," J. Therm. Anal. Calorim., 128, 1711-1717.

Liu, X. and Gu, Y. (2001). "Effects of molecular structure parameters on ring-opening reaction of benzoxazines," Sci. China, 44, 553-560.

Ma, H.X., Zhao, C., Qiu, J.J., Liu, Y. and Liu, C.M. (2017). "Synthesis of branched benzoxazine monomers with high molecular mass, wide processing window, and properties of corresponding polybenzoxazines," J. Appl. Polym. Sci., 134, 1-10.

Macosko, C.W. and Miller, D.R. (1976). "New derivation of average molecular weights of nonlinear polymers," Macromol., 9, 199-206.
Miller, D.R. and Macosko, C.W. (1976). "A new derivation of postgel properties of network polymers," Rubber Chem. Technol., 49, 1219-1231.

Miller, D.R. and Macosko, C.W. (1978). "Average property relations for nonlinear polymerization with unequal reactivity," Macromol., 11, 656-662.

Ning, X. and Ishida, H. (1994). "Phenolic materials via ring-opening polymerization of benzoxazines: effect of molecular structure on mechanical and dynamic mechanical properties," J. Polym. Sci., 32, 921-927.

Rishwana, S.S., Pitchaimari, G. and Vijayakumar, C.T. (2016). "Studies on structurally different diamines and bisphenol benzoxazines: synthesis and curing kinetics," High Perform. Polym., 28, 466-478.

Rucigaj, A., Alic, B., Krajnc, M. and Sebenik, U. (2015). "Curing of bisphenol A-aniline based benzoxazine using phenolic, amino and mercapto accelerators," Express Polym. Lett., 9, 647-657.

Shukla, S., Tripathi, M., Mahata, A., Pathak, B. and Lochab, B. (2016). "Kinetics behind a strategy for modulation of sustainable benzoxazines: experimental study and its theoretical verification," Macromol. Chem. Phys., 217, 1342-1353.

Soto, M., Hiller, M., Oschkinat, H. and Koschek, K. (2016). "Multifunctional benzoxazines feature low polymerization temperature and diverse polymer structures," Polymers, 8,1-14.

Spontón, M., Estenoz, D., Lligadas, G., Ronda, J.C. and Galia, M. (2012). "Synthesis and characterization of a hybrid material based on a trimethoxysilane functionalized benzoxazine," J. Appl. Polym. Sci., 126, 1369-1376.

Spontón, M., Larrechi, M.S., Ronda, J.C. and Galia, M. (2008). "Synthesis and study of the thermal crosslinking of bis(m-aminophenyl) methylphosphine oxide based benzoxazine," J. Polym. Sci. Part A Polym. Chem., 46, 7162-7172.

Wang, H., Zhu, R., Yang P. and Y. Gu, (2016). "A study on the chain propagation of benzoxazine," Polym. Chem., 7, 860-866.

Wang, Y.X. and Ishida, H. (2000). "Synthesis and properties of new thermoplastic polymers from substituted 3,4-Dihydro-2H-1,3-benzoxazines," Macromol., 33, 2839-2847.

Received: May 28, 2018

Sent to Subject Editor: May 5, 2019

Accepted: September 27, 2019

Recommended by Subject Editor M. Luján Ferreira 